\title{
Systematic Evaluation of Arduino Open Source for Automation and Control of Chemical Processes
}

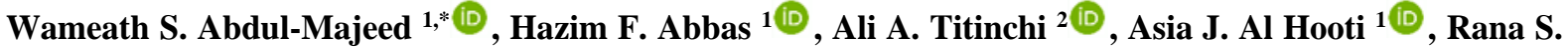 \\ Al Nanwari ${ }^{1}$, Rakan R. Al Najar ${ }^{1}$ \\ 1 Department of Chemical and Petrochemical Engineering - University of Nizwa, Nizwa, PC616, Oman \\ 2 Department of Electrical and Computer Engineering - University of Nizwa, Nizwa, PC616, Oman \\ * Correspondence: wameath@unizwa.edu.om,wameath@yahoo.com;
}

Scopus Author ID 54402262400

Received: 16.10.2020; Revised: 14.11.2020; Accepted: 16.11.2020; Published: 21.11.2020

\begin{abstract}
Inceptions for chemical process automation are presented in this study. A chemical process demonstrated by neutralization reaction was designed, built, and tested experimentally towards evaluating automation and control algorithms through the Arduino Mega platform. The main objective parameter in this work was selected to be the product $\mathrm{pH}$ value, which was evaluated based on several scenarios that targeted various changes in direct and indirect effects. Two main branched ideas were investigated in this study; the first was dealt with the application of Arduino board in the automation of chemical process; the second was dedicated to studying integration of Arduino board in controlling the targeted $\mathrm{pH}$ parameter in the product side. Upon examining different automation scenarios, an algorithm was developed to approach the product quality of specific $\mathrm{pH}$ and temperature efficiently. The automation algorithm was further developed by integrating the process dynamics and control concepts towards speeding up the $\mathrm{pH}$ set point's reach. To make this happen, the pump's speed was corrected and tuned based on the feedback signal from the $\mathrm{pH}$ sensor. Consequently, the setpoint was reached in shorter periods, attaining considerable savings in time $(\approx 35 \%)$. Based on the study outcomes, it is believed that Arduino open source is a challenging and promising low-cost platform, proved useful for mimicking control and automation of chemical processes.
\end{abstract}

Keywords: chemical process control and automation; open source; Arduino Mega; pH sensor; temperature sensor; DC motor pump.

(C) 2020 by the authors. This article is an open-access article distributed under the terms and conditions of the Creative Commons Attribution (CC BY) license (https://creativecommons.org/licenses/by/4.0/).

\section{Introduction}

Since founded by Ivrea Interaction Design Institute, the Arduino platform has become a popular microcontroller used in various fields. Arduino is an open-source electronics platform providing highly reliable, easy-to-use hardware and software in a broad spectrum of applications. This improved electronics platform has attracted many people over the years, beginners and experts of different ages and qualifications, and has been interacted as a core unit of the bulk of projects starting from learning purposes and ending with complex scientific instrumentation. Arduino board is based on the ATmega328 microcontroller unit (MCU) with a variety of interfaces. In addition to availability at low cost, the processor can be programmed using a customized based programming language [1-2]. As of the current development level, the Arduino platform was tagged with essential features such as (1) ability of the board analog and digital input/output ports to process signals from different sources (e.g., temperature and pressure sensors whose output quantity is voltage; thermocouple amplifiers and $\mathrm{pH}$ meters with 
digital output); (2) availability of various types of digital sensors which can be used for measuring various physical parameters; (3) availability of customized boards for specific applications such as printed circuit boards (e.g., signal amplifier and AD converter which contains the sensor itself with other circuits); (4) utilizing the digital outputs of such cards for demonstration purposes (e.g., LCD display); (5) most important is the data management; in which the signals measured can be stored on an SD card or sent to a PC via the USB port [13]. Though these remarkable features, a debate is still rising on the possible emergence of a low-cost Arduino platform as a surrogate to a high-cost programable controller in industrial applications, which requires thorough and deep investigations. In this sense, a comparison between a real-time industrial controller with Arduino was reported by D. Renker [4], in which he identified Arduino board as a programmable logic controller PLC works with simple basic analog automation functions and often uses a PI loop as the control algorithm (doesn't need derivative to read the signal from the measuring device and adjusts the control device to reach and maintain the setpoint). A general opinion on the extent of usage of the developed opensource was based on the fact that Arduino may functions properly should the right program is written to match the application. However, the source may commonly suit regulating the motor's speed or modulating a temperature control loop, but not so good for many other applications.

Only a few publications discussed the application of the Arduino platform in chemistry fields; most of them were dedicated to developing student's teaching and learning. Specifically, Kubinova and Sleger $[1,8]$ used the term ChemDuino to present some of the Arduino platform's capabilities to improve chemistry teaching and to learn in the school environment. In other direction, Arduino board was utilized in lab automation as reported by G. Mabbott [3] in controlling a thermal cycler, Cao et al. [5] in constructing inexpensive electronic burett, and recently by M. Sadegh-Cheri [6] and A. Samokhin [7] in the fabrication of spin coater and syringe pump, respectively. Pereira Filho et al. [9] evaluated the dynamic simulation performance in Arduino for an elementary first-order reaction in a batch reactor in other investigations. They concluded that using Arduinos boards is feasible for solving ordinary differential equations through numerical solutions even with concentrated parameters systems. Moreover, the Arduino platform was used to provide open-loop control of a digital peristaltic pump for studying lymphatic biomechanics in vitro [10].

In the chemical engineering sectors, recent publications discussed Arduino's use in controlling the temperature in a fixed bed reactor [11] through adaptive sliding mode control [12-13]. Other publications discussed the application of Arduino for general monitoring and automation purposes [20-23].

In the current study, we aimed at evaluating the Arduino platform, in terms of automation and control functions, for a chemical process represented by a reaction between acid and base. It is well known that the control of $\mathrm{pH}$ in such a process is considered a challenging task due to several complexities in the reaction path (e.g. nonlinearity of the titration curve). The study aimed to investigate the open-source responses towards controlling the $\mathrm{pH}$ value in a wide selected range. For this purpose, $\mathrm{HCl}$ and $\mathrm{NaOH}$ were chosen as reactants, which are both strong. Thus, their titration curve is known to be very steep near the equilibrium and, therefore, is considered a complicated process to control even by utilizing industry-standard types of controllers. So, this study's outcomes would draw a future picture for the possible application of Arduino open-source in the chemical industry. 


\section{Materials and Methods}

Aqueous solutions of various $\mathrm{pH}$ values in the range (2-13) were prepared using standard hydrochloric acid and sodium hydroxide pellets purchased from Merck and ultra-pure distilled water. The system was examined by applying 2 selected solutions as acid and base towards reaching a specified $\mathrm{pH}$ set point. An experimental setup was built in the current study composed of tanks (glassware's: 2 laboratory conical flasks of $1000 \mathrm{~mL}$ capacity and 3 laboratory beakers of $500 \mathrm{~mL}$ capacity), 5 peristaltic pumps, silicone tubes, and joints, reaction media represented by tubular section, agitator, and various kinds of sensors such as ultrasonic level sensor, temperature sensor, and $\mathrm{pH}$ sensor. The setup is illustrated in Figure 1. This study's examined setup was intended to resemble a full chemical process starting from feeding the reactants to the reaction stage and ending with the final product checkup. Number five glass containers of different capacities were used in this study for various purposes. The first and second containers (to the left of Figure 1) were used as a feed tank for the acid and base solutions, respectively. The acid and base solutions were fed through the peristaltic pumps 1 and 2 to a helical reaction coil composed of 4 loops (silicon tubular section). The reason behind using the helical section was to enhance the mixing through the elaborated secondary flow (a pair of symmetric vortices is formed on the cross-sectional plane due to the centrifugal force), and hence, resulting in higher mass and heat transfer rates [14]. The stream leaving the reaction coil is delivered to tank 3 , which presents an intermediate stage equipped with an agitator, $\mathrm{pH}$, and temperature sensors. The $\mathrm{pH}$ and temperature of the product are being checked.

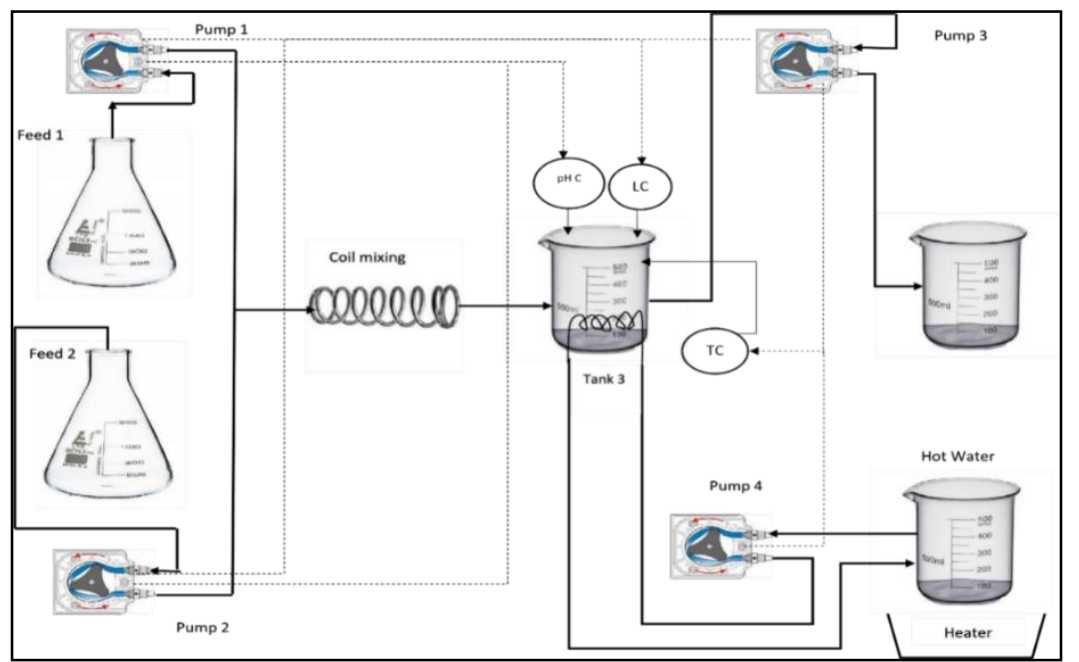

Figure 1. A schematic diagram for the experimental setup.

Should both $\mathrm{pH}$ and temperature values of the product solution have reached the set points, the product is transferred to the final product tank through pump number 3 . The product's temperature in the intermediate tank 3 is tuned through a hot water stream passing through a copper coil ( $4 \mathrm{~mm}$ ID). Figure 1 shows that the hot water tank is being heated to the set temperature by a plated heating magnetic stirrer (Thomas scientific). All peristaltic pumps were selected with a feeding rate $80 \mathrm{ml} / \mathrm{min}$ and powered by a DC power supply (0-30 volt) purchased from Engineering village/Muscat. It's worth mentioning that all peristaltic pumps, silicon tubes (6 mm ID), and the pipe joints used in the setup were purchased from Williamson pumps Ltd./UK. The automation and control of the setup were done through the Arduino Mega board. A standard tool kit for Arduino board, including breadboard, LEDs, wires, resistors (high value), and other accessories, was used to build the electronic circuit in this work. Three 
sensors in the setup located in the intermediate tank were $\mathrm{pH}$ sensor (E-201-C), ultrasonic level sensor (HC-SR04), and temperature sensor (DS18B20, a digital sensor for temperature measurement and is connected to Arduino via the so-called 1-wire bus using one of the digital pins of such Arduino). The signals were transformed to the board through wires connected via a breadboard. The $\mathrm{pH}$ readings were monitored through LCD and recorded and displayed on the computer screen throughout the processing time. Number 4 LEDs with green light were used to enlighten the pump in operation, whereas the other 4 LEDs with orange light were used to indicate the off state. Figure 2 illustrates a picture for this study's whole setup, including the control room, where the Arduino board and the breadboard, and other electrical circuit components were located. Figures 3 and 4 illustrate the wiring diagram for the controls and LEDs, respectively. The operational logic program was written using standard Arduino integrated development environment (IDE) programming code in the study's automation part. The algorithm flowchart for the automation and control logic is shown in appendix 1 (Supplementary file, Figures S1-S3).

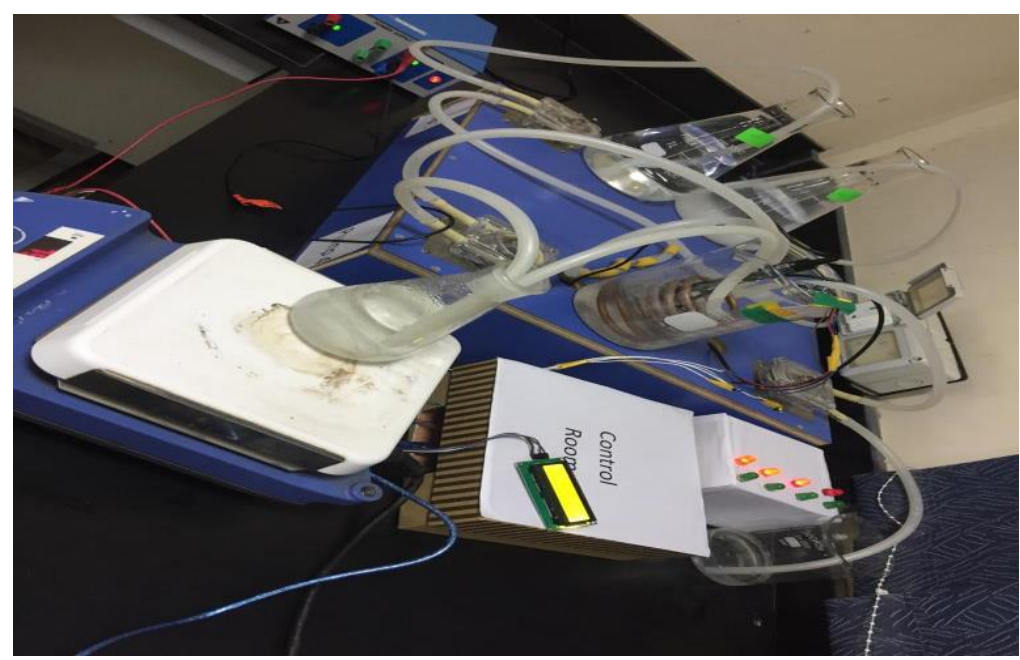

Figure 2. Picture illustrates the whole experimental setup.

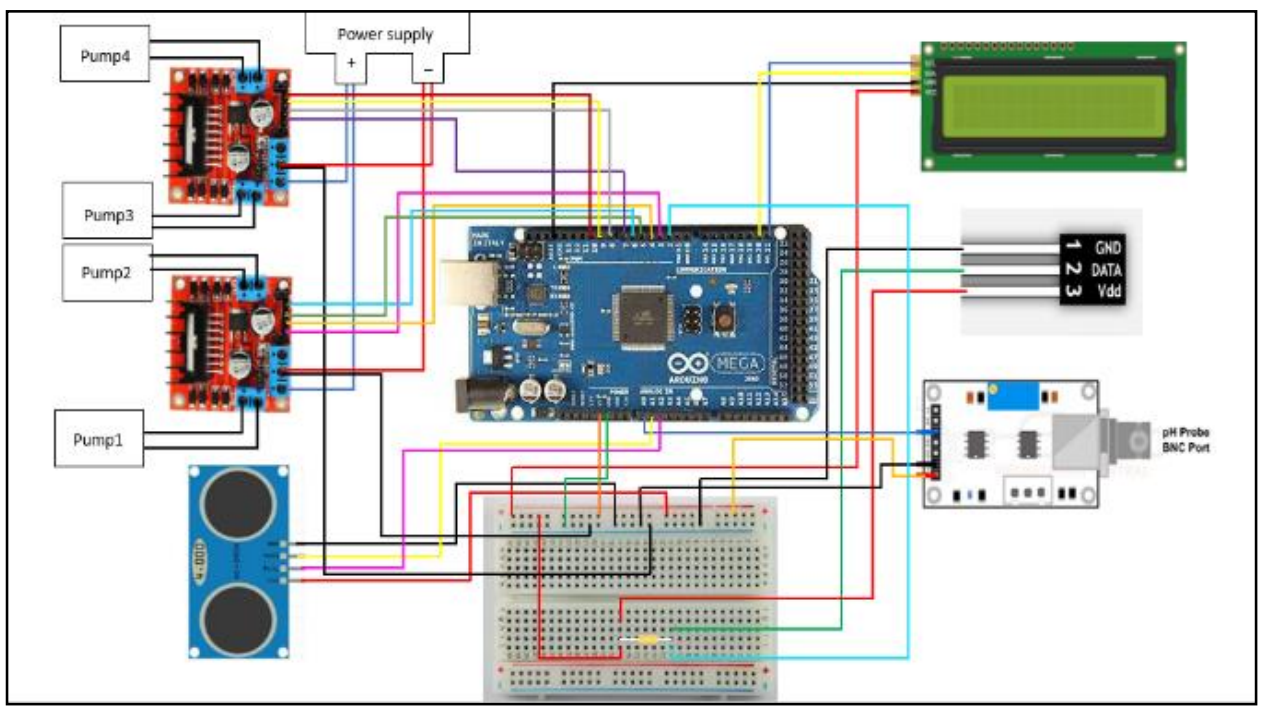

Figure 3. Schematic illustrates the wiring diagram for the whole experimental setup. 


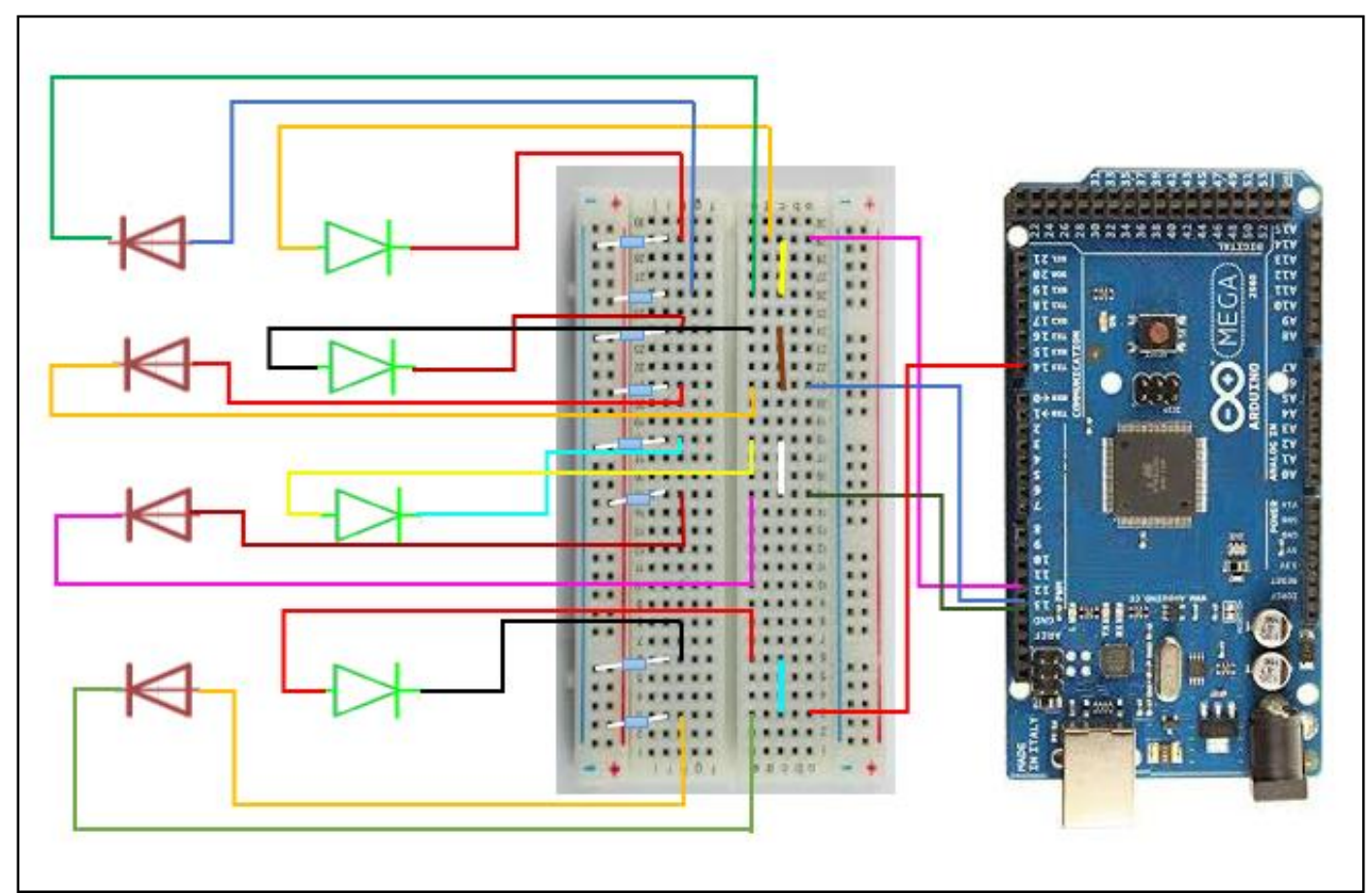

Figure 4. Schematic illustrates the wiring diagram for the LEDs used in the setup.

\section{Results and Discussion}

As previously denoted, this study was dedicated to investigating the application of Arduino open-source for automation and control of the chemical process, as shown in the following details:

\subsection{Application of Arduino board for automation of the chemical process.}

In this part, an experimental approach composed of 13 experiments based on a secondorder central composite rotatable design plan [15] considering five levels of 2 independent variables was adopted to examine the proposed scheme for system automation. The benefits of using the central composite rotatable technique are: minimizing the total number of experiments, help to get the best response surface, help to analyze and sort data easily, and find efficient results in a simple statistical way [16]. This test aimed to contemplate the feasibility of applying the automation of a chemical process at the first level of complexity targeting one independent variable. A target of producing an aqueous solution at a specific set point of $\mathrm{pH}$ value was set for this test. The adopted plan is shown in Table 1. The base solution (feed stream 2 , $\mathrm{pH}$ value in the range 8-12) and the value of the targeted product set point in the range 5-9 were selected to be the two independent variables in this process.

Moreover, the $\mathrm{pH}$ of the acidic solution (stream 1) was fixed at a value 3. In contrast, the time required to reach the $\mathrm{pH}$ set point was measured in each experiment and considered to be the objective function in this process. It should be indicated that the time recorded for each experiment in this set indicates the period elapsed from time zero until approaching the product $\mathrm{pH}$ set point. It should also be indicated that the readings of standard laboratory instruments calibrated all sensors used in the setup. For instance, the $\mathrm{pH}$ sensor was calibrated using a benchtop $\mathrm{pH}$ device from Hanna and applying standard acidic and basic solutions.

Upon conducting the experimental set, an empirical model, presented in Table 2, was depicted for the examined system with a $15.4 \%$ mean absolute error. $Y$ is the objective function, while $x o$ is a dummy variable with the value $(+1)$, whereas $x_{1}$ and $x_{2}$ represents the 
adopted variables' code values ( $\mathrm{pH}$ values of the base solution, stream 2 , and the targeted product set point, respectively). The code values were examined in the range $(-1.414,-1,0$, $+1,+1.414)$, as shown in Table 1 . $B_{i}$ represents the coefficients of the linear terms $\delta y / \delta x_{i}$ whereas $B_{i i}$ and $B_{i j}$ represent the coefficients of the quadratic terms $\delta^{2} y / \delta_{x_{i}}^{2}$ and the cross product terms $\delta^{2} y / \delta_{x_{i}} \delta_{x_{j}}$ that refers to the interaction effects.

Table 1. The experimental plan for system automation.

\begin{tabular}{l|c|c|c|c|c} 
& \multicolumn{2}{|c|}{ Variable1 } & \multicolumn{2}{c}{ Variable2 } & Results \\
\hline \multirow{2}{*}{\begin{tabular}{l} 
No. \\
\cline { 3 - 5 }
\end{tabular}} & Code & Real & Code & Real & $\begin{array}{c}\text { Measured time to reach } \\
\text { the setpoint, sec }\end{array}$ \\
\cline { 2 - 5 } & $\mathrm{X}_{1}$ & $\begin{array}{c}\text { Stream 2 (basic } \\
\text { solution) } \mathrm{pH}\end{array}$ & $\mathrm{X}_{2}$ & $\begin{array}{c}\text { Setpoint, } \\
\mathrm{pH} \text { of the product }\end{array}$ & 279 \\
\hline $\mathbf{1}$ & -1 & 9 & -1 & 6 & 1008 \\
\hline $\mathbf{2}$ & +1 & 11 & -1 & 6 & 850 \\
\hline $\mathbf{3}$ & -1 & 9 & +1 & 8 & 349 \\
\hline $\mathbf{4}$ & +1 & 11 & +1 & 8 & 211 \\
\hline $\mathbf{5}$ & -1.414 & 8 & 0 & 7 & 416 \\
\hline $\mathbf{6}$ & +1.414 & 12 & 0 & 7 & 615 \\
\hline $\mathbf{7}$ & 0 & 10 & -1.414 & 5 & 270 \\
\hline $\mathbf{8}$ & 0 & 10 & +1.414 & 9 & 371 \\
\hline $\mathbf{9}$ & 0 & 10 & 0 & 7 & 300 \\
\hline $\mathbf{1 0}$ & 0 & 10 & 0 & 7 & 349 \\
\hline $\mathbf{1 1}$ & 0 & 10 & 0 & 7 & 360
\end{tabular}

Table 2. Empirical formula depicts the automation of the chemical process targeting the product at the $\mathrm{pH}$ set point.

\begin{tabular}{l|l|l|l|l|l}
$Y=B_{0} \cdot x_{0}+B_{1} \cdot x_{1}+B_{2} \cdot x_{2}+B_{11} \cdot x_{1}^{2}+B_{22} \cdot x_{2}^{2}+B_{12} \cdot x_{1} \cdot x_{2} \quad \ldots$. Equation 1 \\
\hline$B_{0}$ & $B_{1}$ & $B_{2}$ & $B_{11}$ & $B_{22}$ & $B_{12}$ \\
\hline 330.1442956 & 64.73375 & 83.031 & -2.261893925 & 281.402414 & -307.5
\end{tabular}

A non-linear constrained optimization was implemented for the examined experimental set and indicated that a $\mathrm{pH}$ set point of 7.5 could be approached with the shortest time (198 sec) when applying a $\mathrm{pH}$ value of 9 for the basic solution (stream 2) and a $\mathrm{pH}$ value for the acidic solution (stream 1) of 3 . The above assessment was first done for the system performance and directed us to conduct more thorough investigations for evaluating the time needed to reach the set point. This has been done through another experimental set by fixing the required product $\mathrm{pH}$ set point at 7, while feeding stream 1 (acidic solution) and stream 2 (basic solution) at various values of their $\mathrm{pH}$ range. The results are shown in Figures (5-8). The time was recorded based on the period elapsed from reaching the intermediate tank's targeted liquid level until approaching the product $\mathrm{pH}$ set point.

It's noteworthy that the pumps in this test were switched off through Arduino as soon as approaching the setpoint's value $( \pm 5-10 \%)$. The emulated error is commonly expected for $\mathrm{pH}$ measurements and could be attributed to the measuring device's accuracy.

It can be observed from the results of this test that a shorter time of 7 seconds was recorded for a narrow difference in the $\mathrm{pH}$ values of the acidic and basic streams (Figure 5). The recorded periods increased non-linearly when increasing the $\mathrm{pH}$ range gap (Figures 6 - 7). The highest period was recorded when applying $\mathrm{pH}=2$ for the acidic solution (Figure 8). This indicates a more complex neutralization reaction scheme upon applying a higher acidity solution. It can also be observed as a fluctuation in the recorded values. It could be attributed to the $\mathrm{pH}$ sensor's gain. The gain is defined as a two-port circuit's ability to increase the power or amplitude of a signal from the input to the output port [17]. This fluctuation problem was treated partially in the second part of the study by allowing 10 seconds delay between the 
measurements. Hence, Arduino's pH automation part was implemented successfully in this study with some difficulties attributed to the complexity of neutralization (proton transfer) reaction kinetics, which is known to be influenced by altering $\mathrm{pH}$ conditions during the process [18].

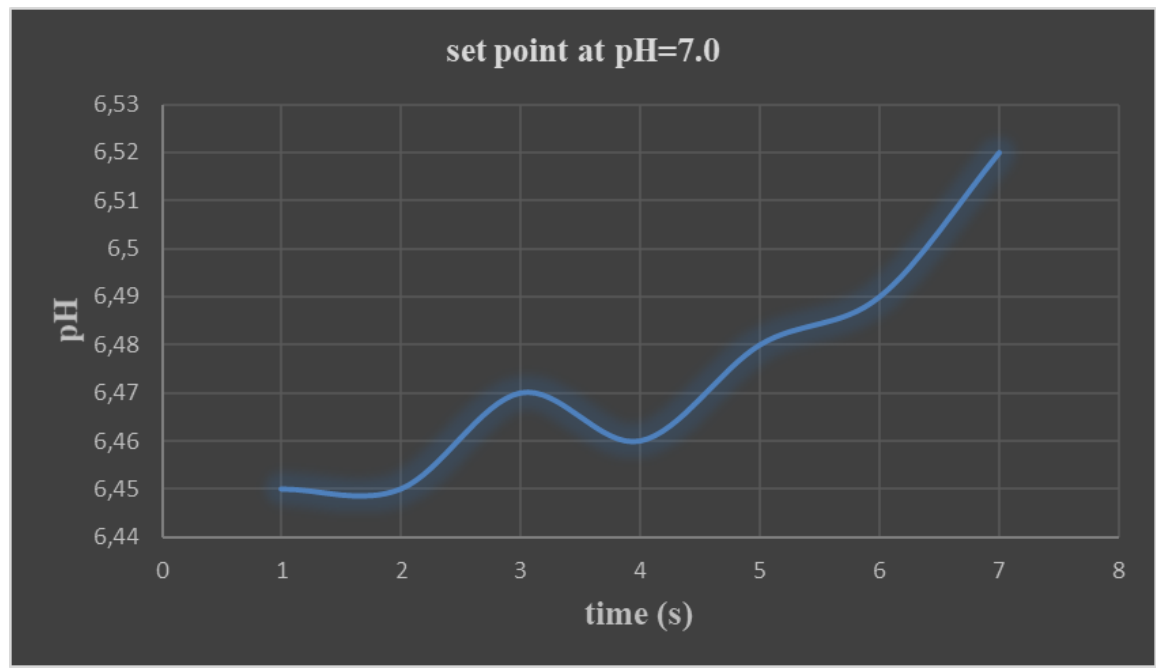

Figure 5. The time elapsed to reach the setpoint when applying the acidic solution $(\mathrm{pH}=5)$ and base solution $(\mathrm{pH}=11)$.

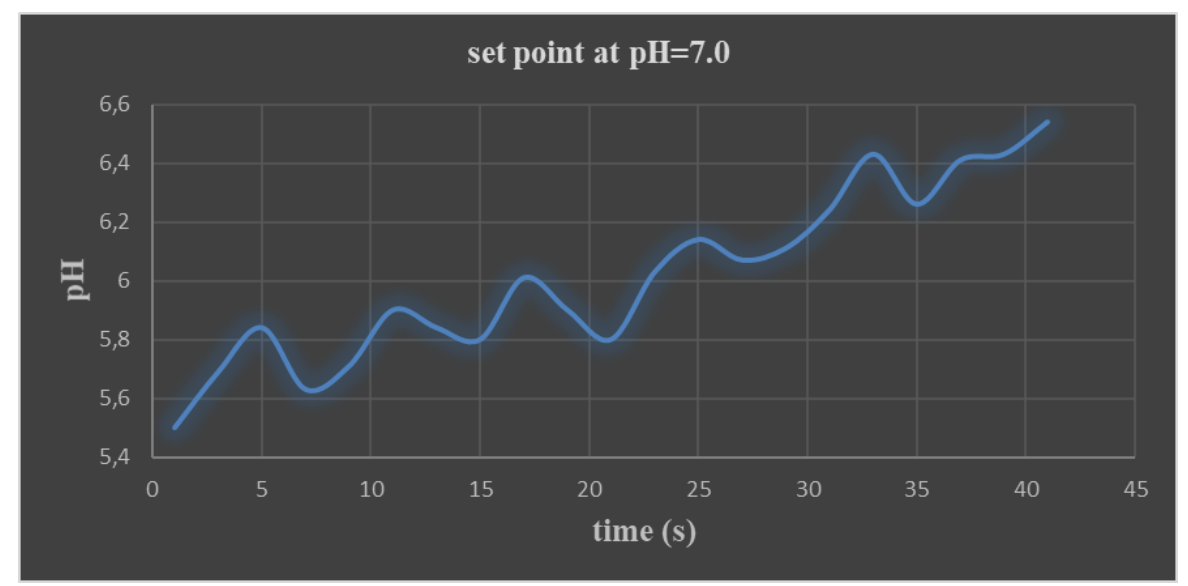

Figure 6. The time elapsed to reach the setpoint when applying the acidic solution $(\mathrm{pH}=3)$ and base solution $(\mathrm{pH}=11)$.

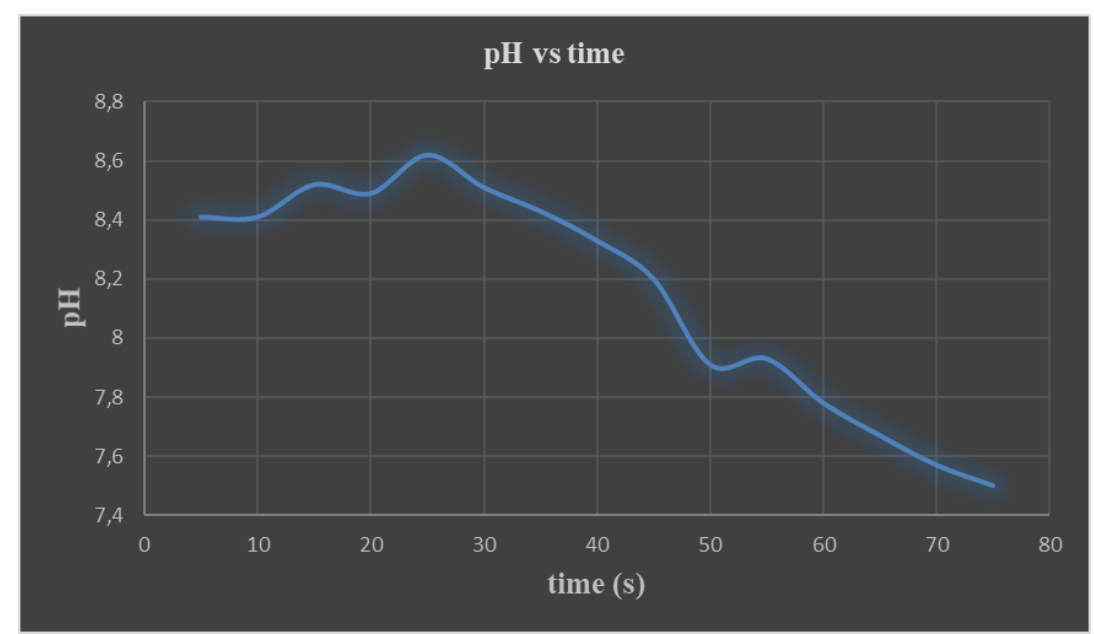

Figure 7. The time elapsed to reach the setpoint when applying the acidic solution $(\mathrm{pH}=3)$ and base solution ( $\mathrm{pH}=12)$. 


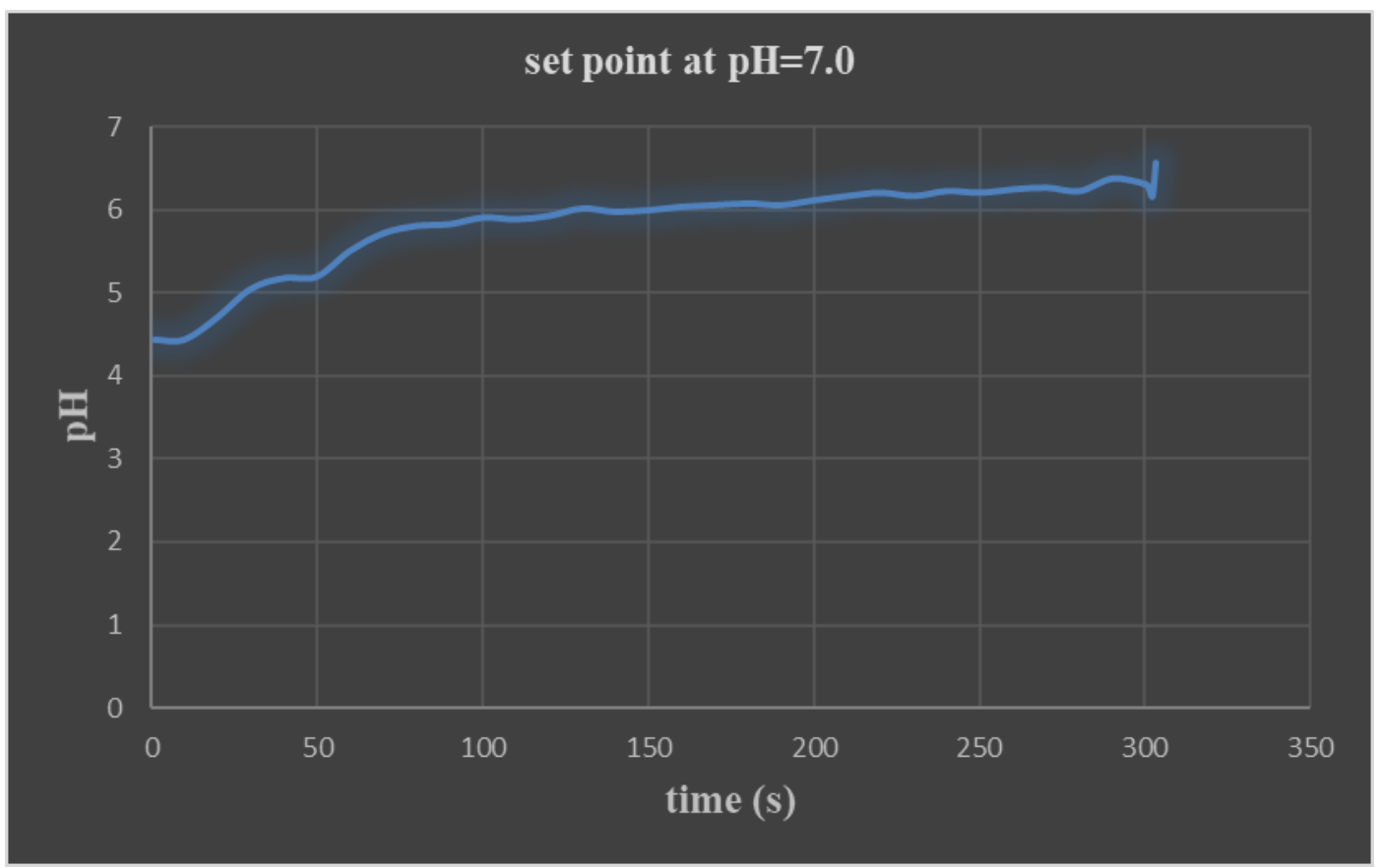

Figure 8. The time elapsed to reach the setpoint when applying the acidic solution $(\mathrm{pH}=2)$ and base solution $(\mathrm{pH}=8)$.

\subsection{Application of Arduino board for the control of $\mathrm{pH}$ in the neutralization process.}

The control of $\mathrm{pH}$ through the Arduino platform was implemented with the same setup by utilizing the feeding tanks ( 1 and 2$)$, the peristaltic pumps (1 and 2), the helical reaction coil, and the intermediate product tank. The stages of the temperature control and the transfer of the solution to the final product tank were excluded in this part of the study. The chemicals for the $\mathrm{pH}$ control process was based on a strong acid/strong base neutralization reaction criteria, and they were: sodium hydroxide (basic solution of 0.05 molarity, $\mathrm{pH}=12.5$ prepared from dissolving 2 grams of $\mathrm{NaOH}$ pellets in 1 liter ultra-pure distilled water); hydrochloric acid (acidic solution of 0.05 molarity, $\mathrm{pH}=1.5$ prepared from diluting $3.2 \mathrm{~mL}$ of $\mathrm{HCl}$ in 1 liter ultrapure distilled water).

This part of the study was dedicated to investigating the possible scenarios towards reducing the time required to reach the $\mathrm{pH}$ set point to the minimum. We processed this objective by controlling the pump speed and relate the function to the offset (the difference between the measured and the setpoint values). The control process was simulated to a closedloop implies that the processor collects input from the device output with the aid of a sensor and uses it to measure the control signal applied to the physical system [19].

In the same principle adopted in the automation part, the Arduino program empowers the pumps 1 and 2 to feed the solutions from tanks 1 and 2 through the reaction helical coil into the intermediate product tank. Upon reaching the liquid level equivalent to $400 \mathrm{~mL}$ in the intermediate product tank, the sensor measures the $\mathrm{pH}$ value. It sends the signal to the controller (Arduino board). Based on the measured offset, the board's signal should empower pump 2 to feed the intermediate tank's basic solution towards tuning the measured $\mathrm{pH}$ based on the set point. A focus on the pump speed control was conducted through several steps: (i) examining different values of the speed of pump 2 (the basic solution pump) seeking the optimum value; (ii) adopting the optimum pump speed from (i) to apply the abovementioned control protocol through finding a relationship with the offset. 
The first step was started by examining various pump speed values (analog signals in the range $150-250$, equivalent to $3-7 \mathrm{~mL} / \mathrm{sec}$ ) in 8 experiments (4 step-up and 4 step-down). Sample of the results at various pump speeds is presented in Figures (9-11). Upon examining the full experimental set, the results have shown a speed of 180 (analog signal, equivalent to 5 $\mathrm{mL} / \mathrm{sec}$ ) is optimum in terms of the attained offset, considering both step-up and step-down experiments, as illustrated in Figure 12. Thus, this pump speed was selected to test the control protocol towards reducing the time needed to reach the setpoint.

In this sense, Figure 13 illustrates the relationship between various pump speed values and the attained offset. The data was fitted exponentially. The produced formula, shown in Figure 13, was introduced in the Arduino program, aiming at tuning the speed based on the calculated offset (measured $\mathrm{pH}$ - set point $\mathrm{pH}$ ). This has been done as per the following logic: The tuning step is being accomplished as soon as an offset value higher than zero is detected. Accordingly, a new pump speed is calculated from the elaborated formula $[y=0.0022$ exp $(0.0298 x)$ ], where $y$ and $x$ represent the offset and the pump speed, respectively. The old value then replaces the corrected pump speed $(\mathrm{x})$ in the program. Hence, the application of the developed logic has fostered reaching the set point with a considerable saving in time. The attained improvement is considered valued as per the logical control principles.

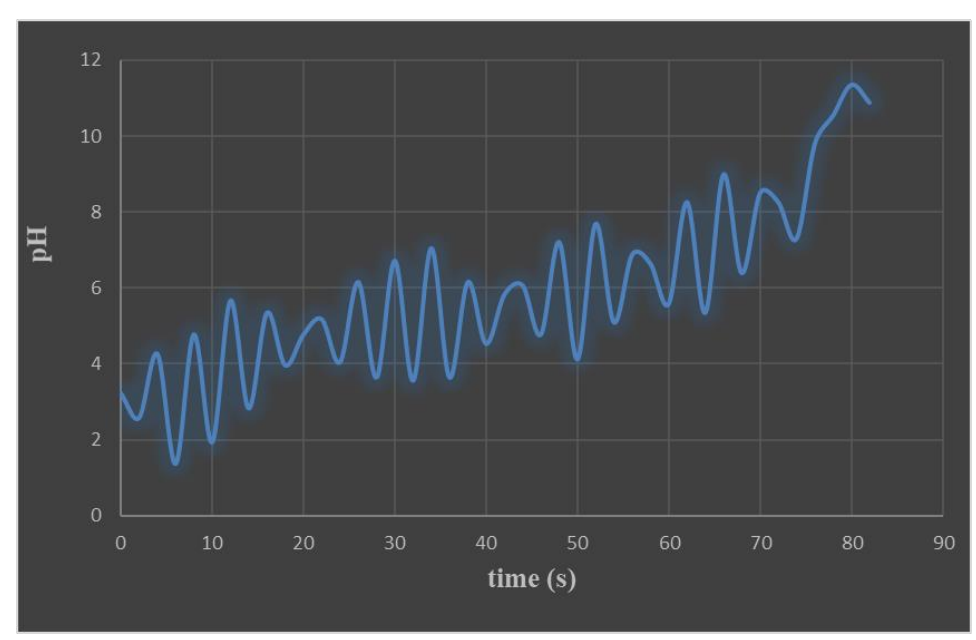

Figure 9. A step-up experiment at speed (3mL/s) from $\mathrm{pH} 3$ to $\mathrm{pH} 11$.

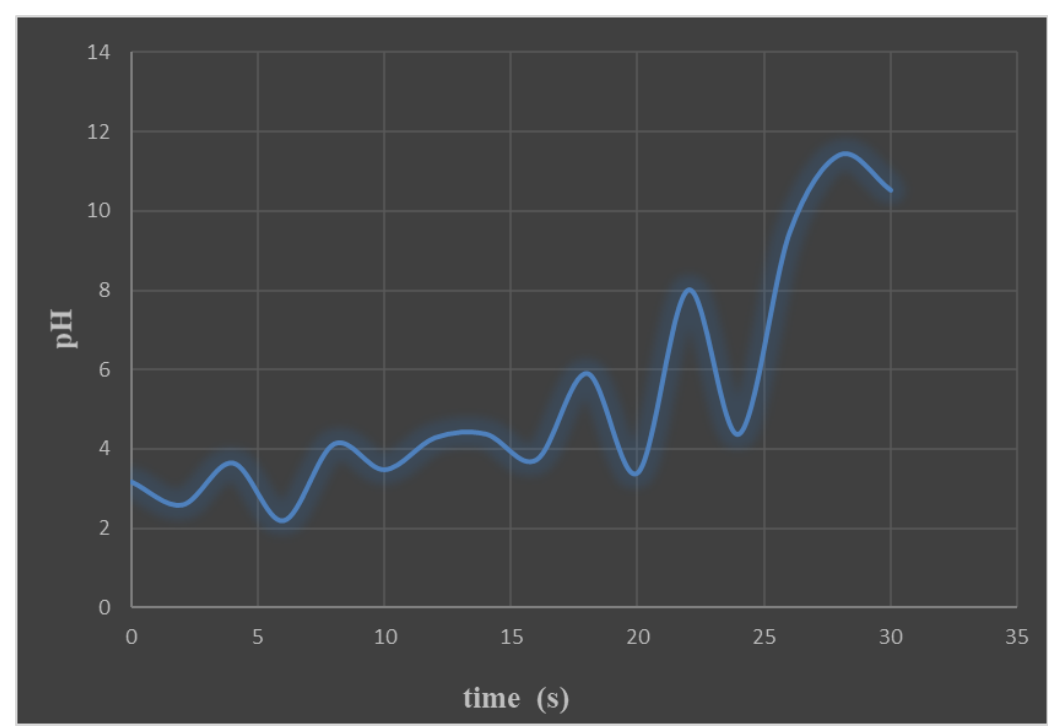

Figure 10. Step up the experiment at speed $(5 \mathrm{~mL} / \mathrm{s})$ from $\mathrm{pH} 3$ to $\mathrm{pH} 11$. 


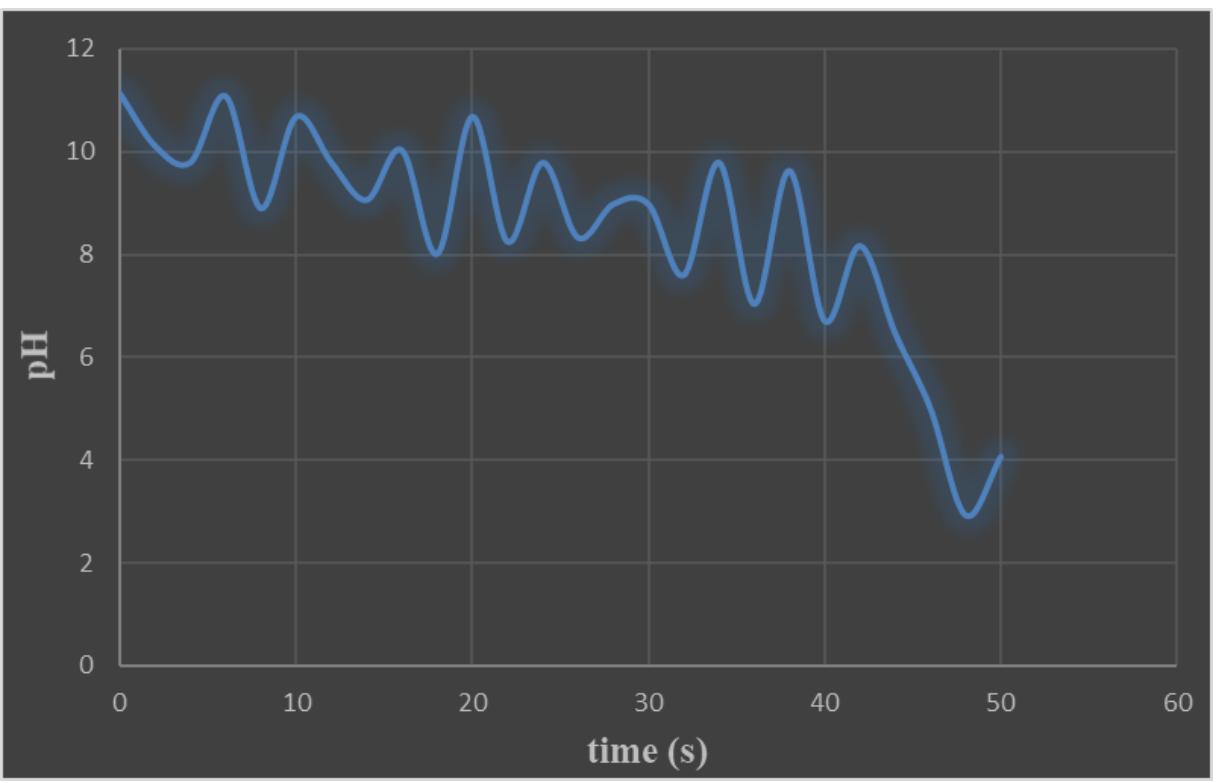

Figure 11. A step-down experiment at speed $(7 \mathrm{~mL} / \mathrm{s})$ from $\mathrm{pH} 11$ to $\mathrm{pH} 3$.

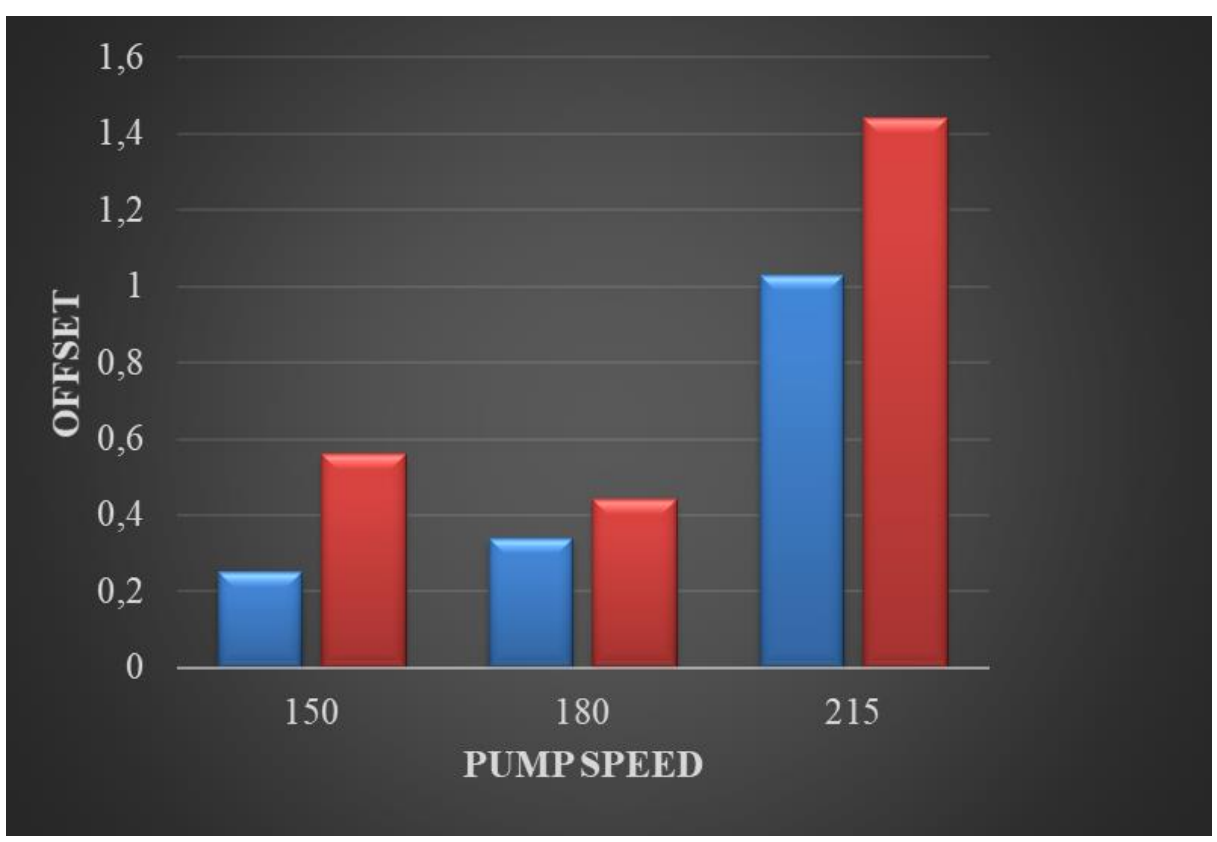

Figure 12. Comparison between the attained offset in various speeds step-up (red color) and step-down (blue color) experiments [pH 3 to $\mathrm{pH} 11$ and vice versa].

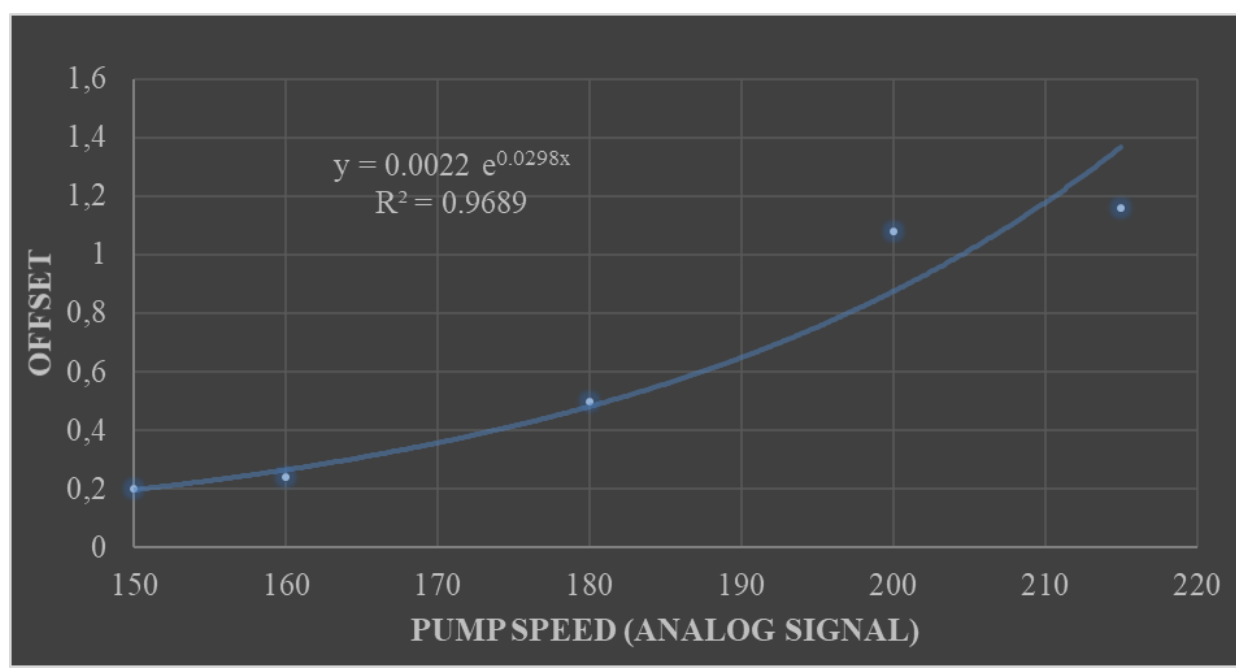

Figure 13. The attained offset vs. pump 2 speed. 


\section{Conclusions}

This study was dedicated to the feasibility of using Arduino open-source for automation and control of the chemical process. An experimental setup was built to resemble a full chemical process from feeding the reactants and ending with delivering the product in the last stage. An automation logic was intended to produce a product at specific set points of $\mathrm{pH}$ and temperature. Upon examining a set of 13 experiments based on the 2nd order central composite rotatable design plan, the results indicated a unique Arduino board's unique response as a first evaluation step towards handling the full automation procedures. However, some difficulties were experienced with and attributed to the gain happened in the electronic circuit. In the second part of the study, a control logic was built and focused on the product $\mathrm{pH}$ control. It is considered the complex kinetics of a neutralization process. The proposed control logic was based on correlating the pump speed with the calculated offset (the difference between the measured and the setpoint values). Several steps were conducted towards this objective, starting with evaluating the optimum pump speed and applying the control program's corrected speed. The developed logic led to a worth saving in the time needed to reach the set point. Thus, this study proposed Arduino's feasible use in automation and control of the neutralization process, a promising start for further in-depth investigations towards emerging this open source in the industry.

\section{Funding}

This research received no external funding.

\section{Acknowledgments}

This research has no acknowledgment.

\section{Conflicts of Interest}

The authors declare no conflict of interest.

\section{References}

1. Kubínová, Š .; Šlégr, J. ChemDuino: Adapting Arduino for Low-Cost Chemical Measurements in Lecture and Laboratory. Journal of Chemical Education 2015, 92, 1751-1753, https://doi.org/10.1021/ed5008102.

2. Arduino Home Page. http://arduino.cc/ Accessed April 2020.

3. Mabbott, G.A. Teaching Electronics and Laboratory Automation Using Microcontroller Boards. Journal of Chemical Education 2014, 91, 1458-1463, https://doi.org/10.1021/ed4006216.

4. Doug Reneker. PLC vs. Arduino for industrial control. https://www.controldesign.com/articles/2017/arduino-vs-plc-for-industrial-control/ Accessed April 2020.

5. Cao, T.; Zhang, Q.; Thompson, J.E. Designing, Constructing, and Using an Inexpensive Electronic Buret. Journal of Chemical Education 2015, 92, 106-109, https://doi.org/10.1021/ed500509p.

6. Sadegh-Cheri, M. Design, Fabrication, and Optical Characterization of a Low-Cost and Open-Source Spin Coater. Journal of Chemical Education 2019, 96, 1268-1272, https://doi.org/10.1021/acs.jchemed.9b00013.

7. Samokhin, A.S. Syringe Pump Created using 3D Printing Technology and Arduino Platform. Journal of Analytical Chemistry 2020, 75, 416-421, https://doi.org/10.1134/S1061934820030156.

8. Kubinova, S.; Slegr, J. ChemDuino-Low Cost System for School Experiments in Chemistry. http://lide.uhk.cz/prf/ucitel/slegrja1/chemduino/index.htm Accessed April 2020.

9. Pereira, R. Dynamic Simulation of Chemical Kinetics in Microcontroller. International Journal of Engineering Research and Applications 2014, 12, 34-38.

10. Kornuta, J.A.; Nipper, M.E.; Dixon, J.B. Low-cost microcontroller platform for studying lymphatic biomechanics in vitro. J Biomech 2013, 46, 183-186, https://doi.org/10.1016/j.jbiomech.2012.09.031. 
11. Pundir, A.S.; Singh, K. Temperature control of real-time identified fixed bed reactor by adaptive sliding mode control equipped with Arduino in Matlab. Asia-Pacific Journal of Chemical Engineering 2019, 14, https://doi.org/10.1002/apj.2297.

12. Sinha, A.; Mishra, R.K. Control of a non-linear continuous stirred tank reactor via event triggered sliding modes. Chemical Engineering Science 2018, 187, 52-59, https://doi.org/10.1016/j.ces.2018.04.057.

13. Amini, M.R.; Shahbakhti, M.; Pan, S. Adaptive Discrete Second-Order Sliding Mode Control With Application to Nonlinear Automotive Systems. Journal of Dynamic Systems Measurement and Control 2018, 140, 1-12, https://doi.org/10.1115/1.4040208.

14. Abdul-Majeed, W. Numerical optimization of the mixing flow in a straight tube, single, and two loops helical coil. Frontiers of Mechatronical Engineering 2018, 1, 1-9, https://doi.org/10.18282/fme.v1i1.601.

15. Cochran, W.G.; Cox, G.M. Experimental Designs. $2^{\text {nd }}$ ed.; Wiley \& Sons: New York, 1992; pp. 382.

16. Abdul-Majeed, W.S.; Al-Riyami, K.O. Activation of peat soil carbon and production of carbon nanostructures using a flying jet cold plasma torch. Environmental Chemistry Letters 2019, 17, 1383-1390, https://doi.org/10.1007/s10311-019-00869-x.

17. Graf, R.F. Modern Dictionary of Electronics. $7^{\text {th }}$ ed. Newnes. 1999; pp. 314.

18. Tong, M.L.; Chen, X.M. Chapter 8 - Synthesis of Coordination Compounds and Coordination Polymers. In: Modern Inorganic Synthetic Chemistry (Second Edition). Xu, R.; Xu, Y. Eds. Elsevier: Amsterdam, 2017; pp. 189-217, https://doi.org/10.1016/B978-0-444-63591-4.00008-2.

19. Wright, R.A.; Kravaris, C. Non-linear control of $\mathrm{pH}$ processes using the strong acid equivalent. Industrial \& Engineering Chemistry Research 1991, 30, 1561-1572, https://doi.org/10.1021/ie00055a022.

20. Yanes, A.R.; Martinez, P.; Ahmad, R. Towards automated aquaponics: A review on monitoring, IoT, and smart systems. Journal of Cleaner Production 2020, 263, https://doi.org/10.1016/j.jclepro.2020.121571.

21. González, I.; Calderón, A.J. Integration of open source hardware Arduino platform in automation systems applied to Smart Grids/Micro-Grids. Sustainable Energy Technologies and Assessments 2019, 36, https://doi.org/10.1016/j.seta.2019.100557.

22. Mumtaz, Z.; Ullah, S.; Ilyas, Z.; Aslam, N.; Iqbal, S.; Liu, S.; Meo, J.A.; Madni, H.A. An Automation System for Controlling Streetlights and Monitoring Objects Using Arduino. Sensors (Basel) 2018, 18, https://doi.org/10.3390/s18103178.

23. Pundir, A; Singh, K. Temperature control of real-time identified fixed bed reactor by adaptive sliding mode control equipped with Arduino in Matlab. Asia Pacific Journal of Chemical Engineering 2019, 14, https://doi.org/10.1002/apj.2297. 


\section{Supplementary file}

\section{Automation and the control logic}

The automation logic consists of three programs illustrated by Figures (S1, S2, and S3). The first program controls the level and $\mathrm{pH}$ values. In the second program, monitoring the temperature up to reaching the setpoint is implemented. Program three is allowing the fluid to be discharged from the intermediate tank upon reaching the desired set points.

In program 1, pumps 1 and 2 operate to pump the acid and base, respectively. The ultrasonic sensor reads the intermediate tank's liquid level until reaching the minimum level equivalent to $400 \mathrm{ml}$. Upon reaching the minimum level, both pumps 1 and 2 are stopped. Then the $\mathrm{pH}$ sensor reads the $\mathrm{pH}$ value and compares it with the set point. If the measured $\mathrm{pH}$ is bigger than the setpoint, then pump 1 (acid pump) will operate. If the $\mathrm{pH}$ is measured to be less than the setpoint, then pump 2 (base pump) will operate. Should the measured $\mathrm{pH}$ value be in the set range, the program will move to the second step. In program two, the temperature sensor reads the temperature value for the intermediate product tank's delivered solution. Should the measured temperature be found less than the setpoint, pump 4 will warm the product to the desired temperature. Should the temperature in the intermediate tank is found in the desired range. The solution is pumped to the final product tank through program 3. Otherwise, the signal will be reversed to run program 1 or 2 as needed per the logic.

The control logic is practically resembling the automation logic; however, only program 1 and 2 were adopted while introducing the correlation $y=0.0022 \exp (0.0298 x)$, which is aimed at correcting the pump speed concerning the detected offset [difference between the measured and the setpoint values].

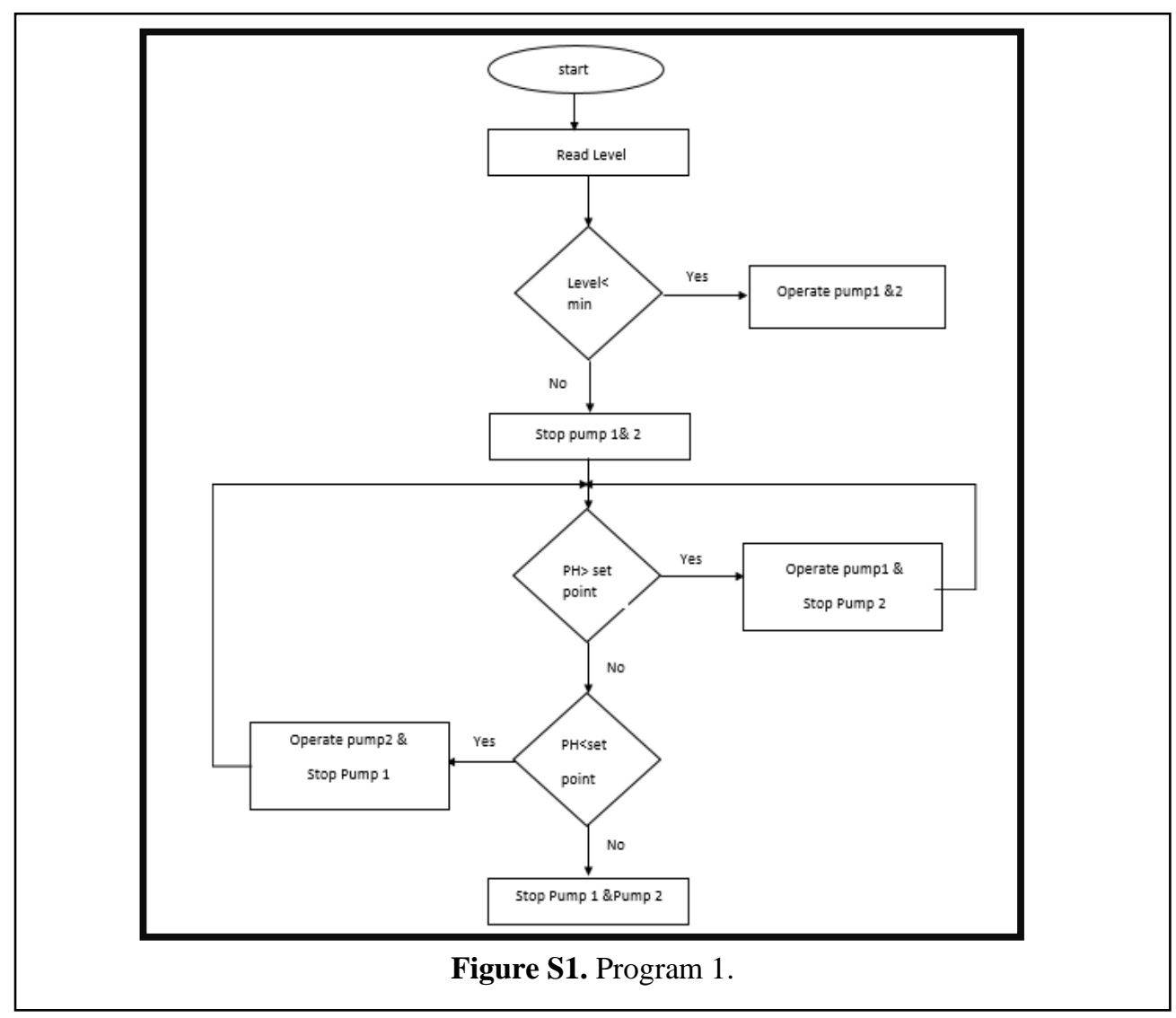




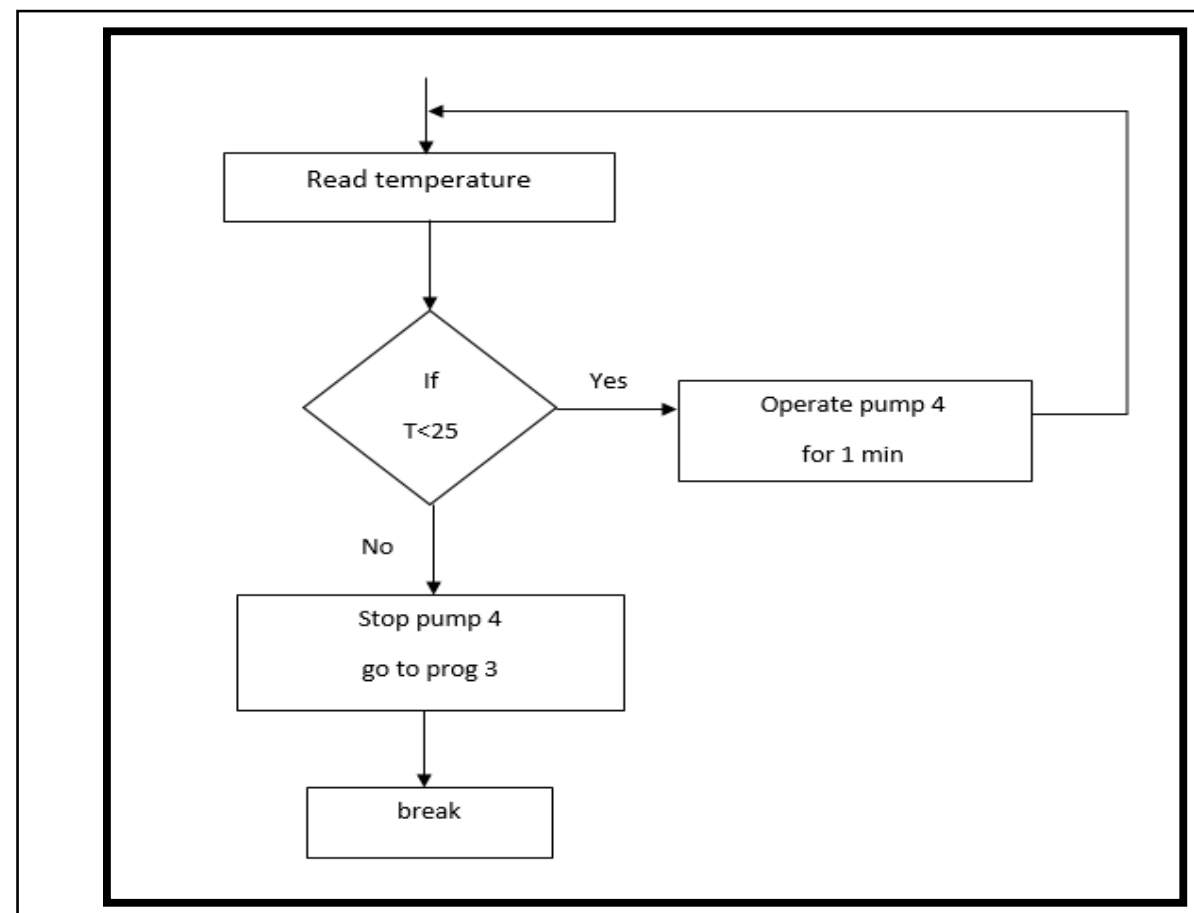

Figure S2. Program 2.

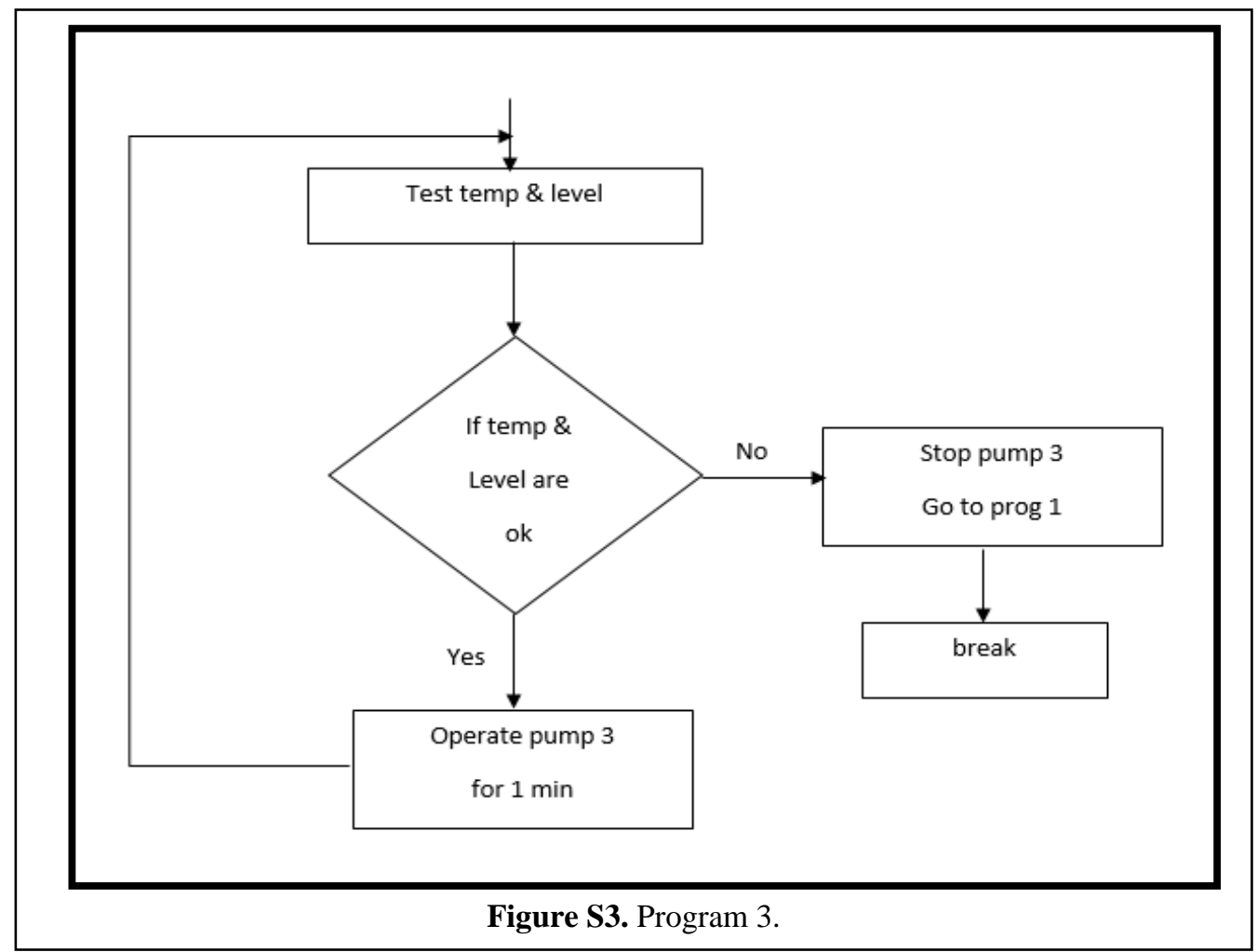

